\section{Lung Segmentation using Active Shape Model to Detect the Disease from Chest Radiography}

\author{
Masoumeh Dorri Giv ${ }^{1}$, Meysam Haghighi Borujeini², Danial Seifi \\ Makrani ${ }^{3}$, Leila Dastranj ${ }^{4}$, Masoumeh Yadollahi ${ }^{5}$, Somayeh Semyari ${ }^{6}$, \\ Masoud Sadrnia7, Gholamreza Ataei ${ }^{8}$, Hamideh Riahi Madvar9*๑
}

\begin{abstract}
Background: Some parametric models are used to diagnose problems of lung segmentation more easily and effectively.

Objective: The present study aims to detect lung diseases (nodules and tuberculosis) better using an active shape model (ASM) from chest radiographs.

Material and Methods: In this analytical study, six grouping methods, including three primary methods such as physicians, Dice similarity, and correlation coefficients) and also three secondary methods using SVM (Support Vector Machine) were used to classify the chest radiographs regarding diaphragm congestion and heart reshaping. The most effective method, based on the evaluation of the results by a radiologist, was found and used as input data for segmenting the images by active shape model (ASM). Several segmentation parameters were evaluated to calculate the accuracy of segmentation. This work was conducted on JSRT (Japanese Society of Radiological Technology) database images and tuberculosis database images were used for validation.
\end{abstract}

Results: The results indicated that the ASM can detect $94.12 \pm 2.34 \%$ and 94.38 $\pm 3.74 \%$ (mean \pm standard deviation) of pulmonary nodules in left and right lungs, respectively, from the JRST radiology datasets. Furthermore, the ASM model detected $88.33 \pm 6.72 \%$ and $90.37 \pm 5.48 \%$ of tuberculosis in left and right lungs, respectively.

Conclusion: The ASM segmentation method combined with pre-segmentation grouping can be used as a preliminary step to identify areas with tuberculosis or pulmonary nodules. In addition, this presented approach can be used to measure the size and dimensions of the heart in future studies.

Citation: Dorri Giv M, Haghighi Borujeini M, Seifi Makrani D, Dastranj L, Yadollahi M, Semyari S, Sadrnia M, Ataei Gh, Riahi Madvar H. Lung Segmentation using Active Shape Model to Detect the Disease from Chest Radiography. J Biomed Phys Eng. 2021;11(6):747-756. doi: $10.31661 /$ jbpe.v0i0.2105-1346.

\section{Keywords}

Active Shape Model; Lung Diseases; Segmentation; Chest; Heart; Diaphragm Radiograph; Radiography

\section{Introduction}

I $\mathrm{n}$ computer vision, segmentation refers to the procedure of partitioning a digital image into several segments (sets of pixels and voxels) to convert an image into separate parts for easier computer calculations [1]. On the other hand, image segmentation is an essential and challenging mission in medical image analysis, particularly with lowquality images, segmentation algorithms can handle ambiguous contex-
${ }^{1} \mathrm{PhD}$, Nuclear Medicine Research Center, Department of Nuclear Medicine, Ghaem Hospital, Mashhad University of Medical Sciences, Mashhad, Iran ${ }^{2} \mathrm{MSc}$, Department of Medical Physics, Isfahan University of Medical Sciences,

Isfahan, Iran

${ }^{3}$ PhD Candidate, Department of Medical Physics and Biomedical Engineering, School of Medicine,

Tehran University of Medi-

cal Sciences, Tehran, Iran

${ }^{4} \mathrm{MSc}$, Department of

Physics, Hakim Sabzevari

Universuty, Sabzevar, Iran

${ }^{5} \mathrm{MSc}$, Department of Allied Medical Sciences, Semnan University of Medical Sci-

ences, Semnan, Iran

${ }^{6} \mathrm{MSc}$, Department of

Physic, Imam Khomeini

International University,

Qazvin, Iran

${ }^{7} \mathrm{BSc}$, Department of Radiology Technology, Rofeideh

Rehabilitation Hospital,

Tehran, Iran

${ }^{8} \mathrm{MSc}$, Department of Radi-

ology Technology, Faculty

of Paramedical Sciences,

Babol University of Medical

Science, Babol, Iran

${ }^{9} \mathrm{MSc}$, Department of Nu-

clear Engineering, Faculty

of Engineering, Science

and Research of Tehran

Branch, Islamic Azad Uni-

versity, Tehran, Iran

*Corresponding author:

Hamideh Riahi Madvar

Department of Nuclear

Engineering, Faculty of

Engineering, Science

and Research of Tehran

Branch, Islamic Azad

University, Tehran, Iran

E-mail: Hamidehriahi-

madvar@gmail.com

Received: 30 May 2021

Accepted: 10 September 2021 
tual clutter, inadequate boundaries, and noise in the image $[2,3]$.

The area can be manually segmented by an expert. Although this method is not used in practice because of operator bias, error, and time-consuming process [4], it is known as the gold standard method $[5,6]$.

Some parametric models are used for lung segmentation include active shape model (ASM), active appearance model (AAM), and pixel classification (PC) [7]. ASM with many internal parameters is a common segmentation method [8] based on shape-learning data to remedy the limitations of deformable models by restricting the deformation of the model to shapes in the acceptable domains. In addition, the ASM model restricts the deformations by learning shape information from pre-segmented form training kits. The primary ASM models were restricted to 2-dimensional spaces; however, 3-dimensional versions of this technique have been introduced recently $[9,10]$. In 3D, ASM models are highly appropriate for segmenting objects with spherical topology, though these models are appropriate for a large category of target shapes, and ASMs are produced by performing Principal Component Analysis (PCA) on some manually delimited, enrolled teaching shapes. This interaction creates a statistical Point Distribution Model (PDM) utilized to compel the deformation of shape models during the image segmentation process. The image segmentation process utilizes an image search algorithm, in which the model is extended and deformed iteratively to fit local image data (like an edge) [1].

The frequency and mortality of lung cancer have increased quickly, with five million passing each year because of environmental problems, tobacco smoking, and other related reasons [11]. Medical imaging modalities such as radiography can detect the lung problem and the exact lung segmentation could lead to better diagnosis [12].

According to the literature, several studies evaluating the ASM ability to segment the chest in radiographic images; however, a grouping of chest images with different methods before segmentation has rarely been investigated in previous studies. Further, lung segmentation in chest radiographs of patients with tuberculosis was not evaluated in previous studies. Therefore, this study aimed to segment lungs using the ASM method with pre-segmentation grouping procedures for detecting lung disease (nodules and tuberculosis) from chest radiography images.

\section{Material and Methods}

This analytical study was conducted on patients' lung images from the JSRT (Japanese Society of Radiological Technology) database (154 pictures contain precisely one pneumonic lung nodule and 93 pictures contain no lung nodule) to segment the lungs, help the radiologist in identifying boundaries, and provide an automatic way for identifying images with visual abnormalities such as enlargement of the heart and diaphragm. The findings were then validated using tuberculosis patients' data (107 images) from the Belarusian tuberculosis database. Furthermore, two experienced radiologists segmented five objects in each image. Figure 1 shows the main image and the corresponding segmentation.

\section{Grouping}

The chest radiograph images were divided into three subgroups based on their different appearances for more accuracy. In terms of details, two abnormalities, i.e. diaphragmatic congestion and heart reshaping were used for grouping instead of just dividing them by their appearance used in a previous study [13]. Our selected abnormalities cause a change in the appearance of the lungs in the radiological images, resulting in identifying the better the segmentation method for new underlying anomalies in the image as a disease.

These classifications are conducted by standard segmented images of the SCR database. Initially, as a standard classification, the dia- 

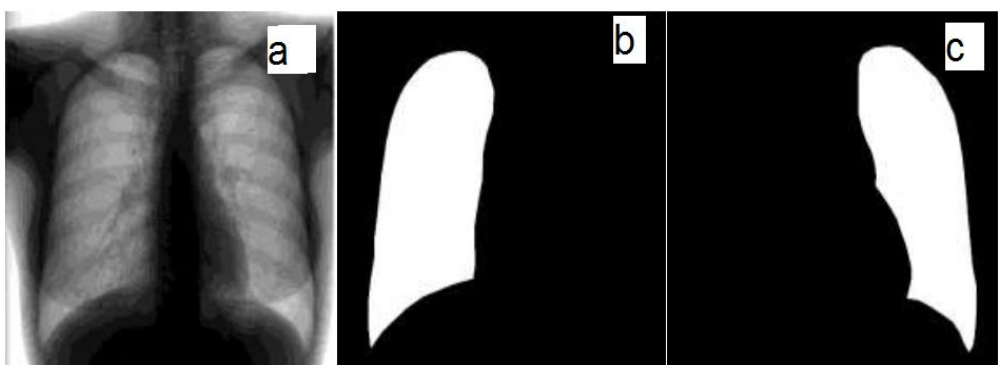

Figure 1: a) Healthy lung image, b) Right lung segmented, and c) Left lung segmented

phragmatic congestion, heart reshaping, and normal lung shape were identified by the specialist, and then three images were selected as the head among each of the mentioned groups. Six classification methods including manual, SVM-manual, Dice similarity, SVMDice, correlation, and SVM-correlation have been applied to this data to identify the best and most accurate method for distinguishing inconsistencies and the most optimal method for increasing the accuracy of ASM code. In the correlation coefficient method, 3 images were selected as heads and the similarity of each image with these images was measured. Therefore, the value of " $r$ " for each image was compared to 3 images of the group obtained using equation 1 [14],

$$
r=\frac{\sum_{m} \sum_{n}\left(A_{m n}-\bar{A}\right)\left(B_{m n}-\bar{B}\right)}{\sqrt{\left(\sum_{m} \sum_{n}\left(A_{m n}-\bar{A}\right)^{2}\right)\left(\sum_{m} \sum_{n}\left(B_{m n}-\bar{B}\right)^{2}\right)}}
$$

where " $\mathrm{r}$ " is the correlation coefficient, $\overline{\mathrm{A}}$ is equal to the average image of header $\mathrm{A}$, and $\overline{\mathrm{B}}$ is the mean of the test image of B. $A_{m n}$ and $B_{m n}$ are $m \times n$ matrices of A and B images. Figure 2 demonstrates the selected head images for each group.

The effect of the iteration number for each point along the variable contour was also examined to obtain the best location for each specific point on the experimental image with the number of $50,70,90$, and 110 repetition points.

Figure 3 illustrates the effect of these repetitions points separately. In the next step, the tuberculosis database images were used as experimental data to determine the effectiveness of this code in detecting lung boundaries. To do this, their group was first identified by the classification method and then segmented by the code trained with the normal data in that group. Finally, the images were placed in the relevant groups with higher " $r$ " values and the secondary grouping was done by the SVM (Support Vector Machine) method. Each of the 93 normal images in the JSRT database was placed in one of these subsets.

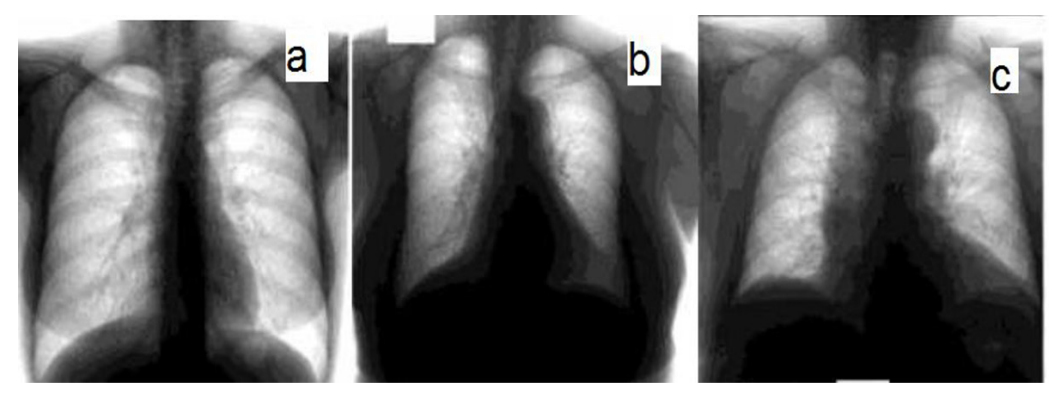

Figure 2: The selected headers for each group a) Healthy lung, b) diaphragmatic congestion, and c) Heart enlargement. 


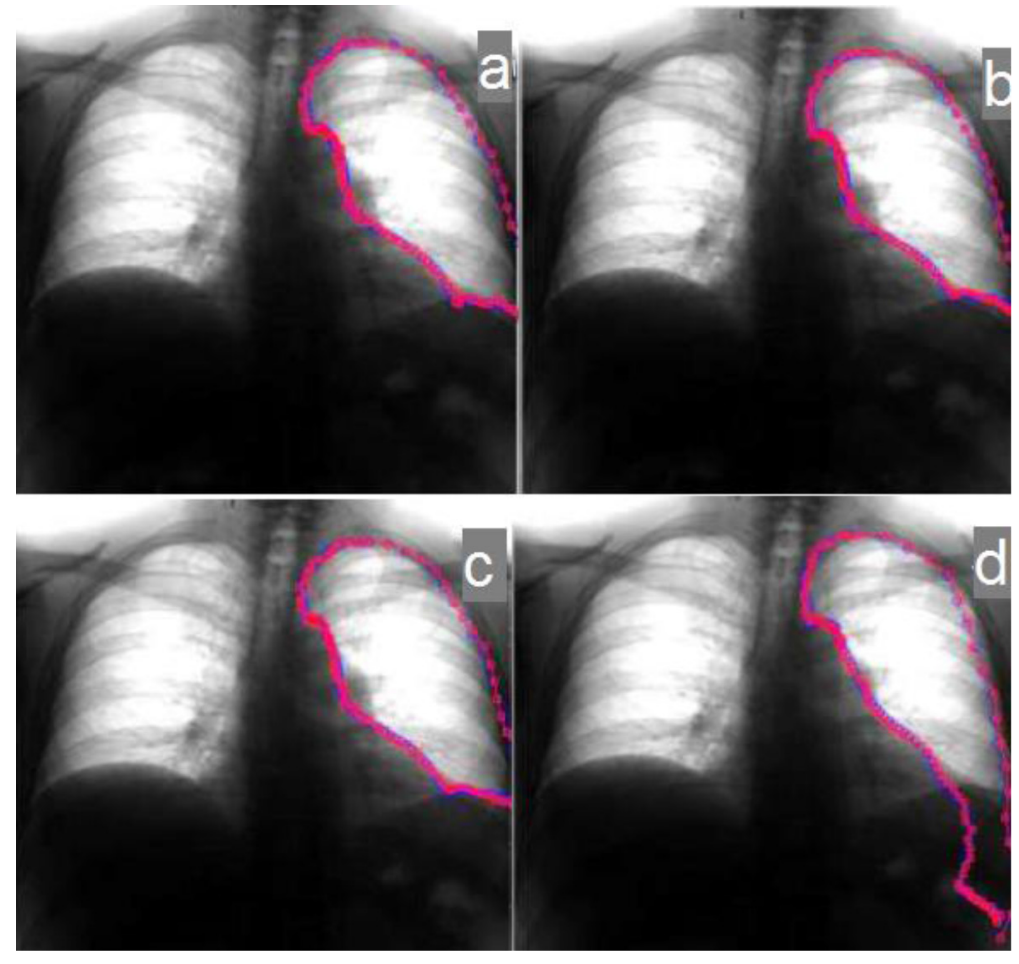

Figure 3: The effect of the repetition number: a) 50, b) 70, c) 90, and d) 110 points.

To evaluate the accuracy of the grouping methods, the SVM code was trained and performed undergoing expert supervision, Dice similarity coefficient, and correlation coefficient. The accuracy of the grouping was measured by a one-way validation method for the mentioned methods. Finally, the most accurate grouping method was selected and then the ASM code was used.

\section{ASM method for segmentation}

The ASM was segmented based on Van Ginneken et al. [15] study. For the training step, it was necessary to specify the contour of the lungs manually so that the contours of all images were drawn at the same time as their standard segmented image by a series of points as major-specific landmarks and another group as minor specific landmark spread around the lung border. The major landmarks must have a fixed number along the entire length of the contour and the minor ones need to be constant between each of the two major landmarks. The location of the major landmarks and the total number of landmarks lead to the accuracy of ASM image segmentation.

Two points (of 21 and 50 points) were considered for the contouring of lungs in the radiographic images; thus, the results of a low and high number of points can be compared. The method used 21 points for segmentation, obtained experimentally as the least number of points resulting in acceptable segmentation. In addition, countering was performed with 50 points for higher accuracy, a method also followed in a previous study [14]. Then the contouring results with 21 and 50 points were compared with each other. After plotting all the contours with 21 and 50 points for both right and left lungs distinctly, these points were used separately as training data for the ASM code. Figure 4 illustrates the results of using 21 and 50-point contours in code training.

The method used to train the code was the cross-validation method; for " $n$ ", the total number of data sets (images), each time $|1-n|$ data were used as training sets and one data 
Active Shape Model in Chest Radiography

as a test set. Therefore, each image would be used as both a training set and a test set. Then accuracy-resulted segmentation was calculated by the Dice similarity coefficient through standard segmented images. The deforming contour sites include the site with the most conformance to the experimental shape, the apex of the lung, the outer wall of the lung, the inner walls of the lung, the farthest point from the heart below the lung, and the closest point to the heart below the lung. The effect of these points on the accuracy of segmentation is seen in Figure 5.

Results

Grouping

The objective of grouping was to divide the a

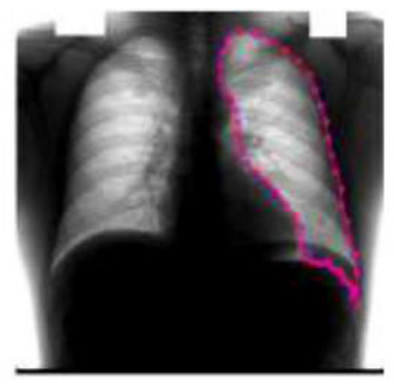

b

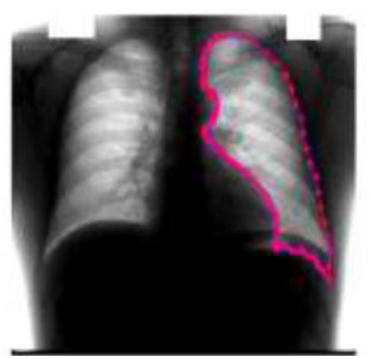

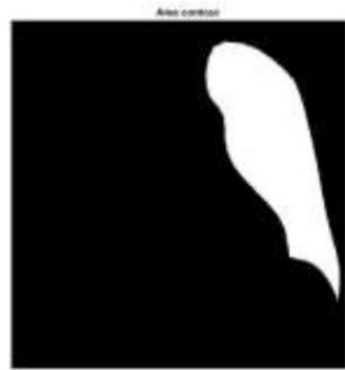

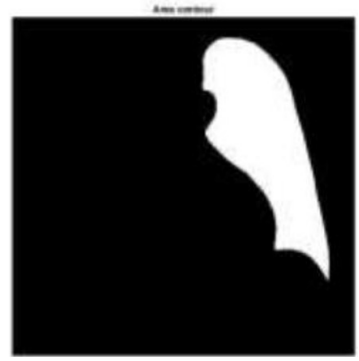

Figure 4: a) Sample image segmented with 21 dedicated points, b) Sample image segmented with 50 dedicated points.
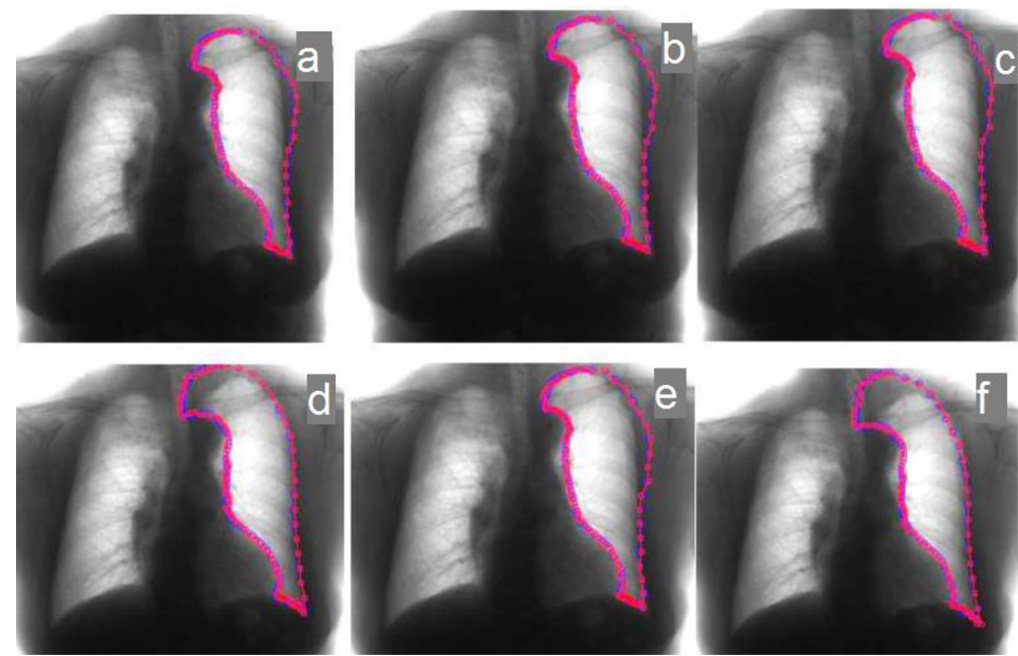

Figure 5: Effect of fixing points located on the accuracy of segmentation. a) The best place visually, b) Apex of the lung, c) Inner wall of the lung, d) Outer wall of the lung, e) The closest point to the heart at the base of the lung, and f) the farthest point to the heart at the base of the lung. 
images into 3 categories: normal, diaphragmatic congestion, and large heart. Since the group classification was performed through a manual method under the supervision of an expert radiologist, it is used for determining the accuracy of segmentation performed by the ASM method with 57, 18, and 18 images, respectively, for normal, diaphragmatic congestion, and large heart.

In addition, 59, 16, and 18 images for the left lung and 47, 17, 29 images for the right lung were used for the Dice similarity coefficient method. Furthermore, for the correlation coefficient (r) method, 63, 14, and 16 images of the three main groups were used for the left lung and 58, 12, and 23 images for the right lung.

The method of grouping using the SVM was trained by groupings with the supervision of a radiologist and a grouping accuracy of 0.799 and 0.655 in the left and right lungs. The SVM by grouping with Dice similarity coefficient in the left lung had a grouping accuracy of 0.828 , and in the right lung had an accuracy of 0.795 and the SVM by grouping with correlation coefficient (r) in the left and right lungs had a grouping accuracy of 0.808 , and 0.806 , respectively.

At last, the images of right and left lungs must be grouped (taking into account both lungs). Therefore, in this study, SVM codes were developed to consider both lungs simultaneously in one image. The total accuracy of these secondary methods, i.e. SVM by grouped images by expert supervision, correlation coefficient, and Dice, was equal to $0.661,0.731$, and 0.2766 , respectively. According to these results, the best secondary grouping method can be considered as the SVM-Dice method with an accuracy of 0.7956 .

\section{Segmentation}

ASM code segmented all images individually after the grouping procedures and the crossvalidation method in each step was applied to increase the accuracy. In this regard, 1-n images were considered as training, and one image was used as a test. In this study, some parameters were investigated for measuring the accuracy of the code such as the effect of repetition number on the final result, the location of the moving contour on the image, and the number of initial contour points created manually for training.

The effect of the point number, designated on the training contour for the left and right lung for Dice similarity grouping technique, was obtained. For left lung, the mean \pm SD (min-max) of accuracy for 21 points segmentation were $0.9016 \pm 0.0504(0.7258-0.9695)$ and $0.9011 \pm 0.0407(0.7338-0.9704)$ for total and dice similarity, respectively. In addition, this value was $0.8714 \pm 0.0441(0.7729$ $0.9579)$ and $0.9066 \pm 0.0412(0.8052-0.9681)$ for 50 points. For segmenting right lung with 21 points, the mean \pm SD (min-max) of accuracy was obtained at $0.9179 \pm 0.0403(0.7836$ $0.9756)$ and $0.9331 \pm 0.0416(0.7530-0.9775)$ for total and dice similarity, respectively, and they were $0.9430 \pm 0.0280(0.8638-0.9845)$ and $0.9494 \pm 0.0289(0.8155-0.9848)$ for 50 points.

The results show the superiority of contours with 50 dedicated points over 21 dedicated points. Improving the accuracy of the segmentation-grouped method compared to the total data segmentation method in a group aims to eliminate the over-training phenomenon and reduce the dependency of the algorithm on the lung volume and background changes in the images.

Due to the superiority of increasing the trained points, only images with 50-point contours were examined. Table 1 illustrates the effect of different fixation locations of deformation contour on the test image with 50 points. The sites examined consist of the apex of the lung, with the most contour and experimental shape, the wall between the lung and the heart, the outer wall of the lung, the point closest to the heart at the base of the lung, and the farthest point from the heart at the base of the lung. These results are for images with 
Table 1: Effect of different fixation locations of deformation contour on the test image (left and right lung) for Dice-50 points.

\begin{tabular}{ccccccc} 
Region & Apex & Best.pos & In.line & Out.line & In.point & Out.point \\
\hline \multirow{2}{*}{ Left lung } & $0.9068 \pm 0.0361$ & $0.9066 \pm 0.0412$ & $0.8942 \pm 0.0550$ & $0.9070 \pm 0.0383$ & $0.8608 \pm 0.0662$ & $0.8627 \pm 0.0666$ \\
& $(0.7643-0.9648)$ & $(0.8052-0.9681)$ & $(0.7120-0.9638)$ & $(0.8058-0.9671)$ & $(0.6466-0.9647)$ & $(0.6635-0.9631)$ \\
\hline \multirow{2}{*}{ Right lung } & $0.9494 \pm 0.0289$ & $0.9508 \pm 0.0288$ & $0.9512 \pm 0.0263$ & $0.9506 \pm 0.0283$ & $0.9423 \pm 0.0325$ & $0.9307 \pm 0.0477$ \\
& $(0.0289-0.9848)$ & $(0.0288-0.9851)$ & $(0.0263-0.9851)$ & $(90.0283-0.9852)$ & $(0.0325-0.9849)$ & $(0.0477-0.9821)$
\end{tabular}

Apex: The apex of the lung, Best.pos: The best position, In.line: Internal line, Out.line: Outer line, In.point: Internal point, Out. point: Outer point

50 training points and grouping with the Dice similarity coefficient method. The overall accuracy of the ASM method was measured by standard segmented images using the Dice similarity coefficient. Based on the results, the best place to select a changeable contour is the apex of the lung and the data are most accurate due to the least movement and change in the apex of the lung in this area.

Tables 2 and 3 show the average, maximum, and minimum accuracy, and the standard deviation for each grouping method with 50 training points and the location of the contour fixation at the apex of the lung. Additionally, the number of repetitions was assessed in Tables
2 and 3.

According to Tables 2 and 3, the high number of repetitions (i.e. 90 and 110 iterations) for the right lung can generally result in similar accuracy with the lower number of iterations; therefore, the lower number of iterations would be more suitable.

The best number of repetitions in the left lung is 70 based on the optimal response time. Among the initial groupings, the Dice similarity coefficient method had the highest accuracy, which was even more accurate compared to the method with expert supervision. After applying the SVM method to each grouping method as a secondary method, the best results

Table 2: The effect of a repetition number on time and segmentation accuracy and comparing different grouping methods in the left lung.

\begin{tabular}{|c|c|c|c|c|c|c|}
\hline & Manual & SVM.manual & Dice & SVM- Dice & $\begin{array}{l}\text { Correlation } \\
\text { ratio }\end{array}$ & $\begin{array}{l}\text { SVM Correlation } \\
\text { ratio }\end{array}$ \\
\hline Itt 50 & $\begin{array}{c}0.09048 \pm 0.0387 \\
(0.7910-0.9712)\end{array}$ & $\begin{array}{l}0.9261 \pm 0.0473 \\
(0.6860-0.9798)\end{array}$ & $\begin{array}{l}0.9068 \pm 0.0361 \\
(0.7643-0.9648)\end{array}$ & $\begin{array}{l}0.09278 \pm 0.0350 \\
(0.8399-0.09795)\end{array}$ & $\begin{array}{l}0.8971 \pm 0.0549 \\
(90.6748-0.9734)\end{array}$ & $\begin{array}{l}0.9254 \pm 0.0394 \\
(0.8025-0.9770)\end{array}$ \\
\hline Time & $43 \min$ & $29.7 \mathrm{~min}$ & $41 \mathrm{~min}$ & $26 \mathrm{~min}$ & $47 \mathrm{~min}$ & $29.8 \mathrm{~min}$ \\
\hline Itt 70 & $\begin{array}{l}0.9048 \pm 0.0406 \\
(0.7893-0.9707)\end{array}$ & $\begin{array}{l}0.9261 \pm 0.0467 \\
(90.6801-0.9797)\end{array}$ & $\begin{array}{l}0.9052 \pm 0.0396 \\
(0.7831-0.9704)\end{array}$ & $\begin{array}{l}0.9280 \pm 0.0352 \\
(0.8305-0.9797)\end{array}$ & $\begin{array}{l}0.8986 \pm 0.0541 \\
(0.6668-0.9722)\end{array}$ & $\begin{array}{l}0.9256 \pm 0.0390 \\
(0.7983-0.9778)\end{array}$ \\
\hline Time & $47 \mathrm{~min}$ & $39.5 \mathrm{~min}$ & $47 \mathrm{~min}$ & $29 \min$ & $52 \mathrm{~min}$ & $41.6 \mathrm{~min}$ \\
\hline Itt 90 & $\begin{array}{l}0.9040 \pm 0.0420 \\
(0.7749-0.9706)\end{array}$ & $\begin{array}{l}0.9169 \pm 0.0451 \\
(0.6201-0.9624)\end{array}$ & $\begin{array}{l}0.9039 \pm 0.0406 \\
(0.7832-0.9696)\end{array}$ & $\begin{array}{l}0.9195 \pm 0.0397 \\
(0.8267-0.9610)\end{array}$ & $\begin{array}{l}0.8918 \pm 0.0576 \\
(0.6801-0.9754)\end{array}$ & $\begin{array}{l}0.9174 \pm 0.0486 \\
(0.8139-0.9612)\end{array}$ \\
\hline Time & $54.5 \mathrm{~min}$ & $50.4 \mathrm{~min}$ & $56.5 \mathrm{~min}$ & $40.1 \mathrm{~min}$ & $62 \mathrm{~min}$ & $55 \mathrm{~min}$ \\
\hline Itt 110 & $\begin{array}{l}0.9003 \pm 0.0427 \\
(0.7495-0.9722)\end{array}$ & $\begin{array}{l}0.9125 \pm 0.0479 \\
(0.9028-0.9514)\end{array}$ & $\begin{array}{l}0.9044 \pm 0.0433 \\
(0.7112-0.9640)\end{array}$ & $\begin{array}{l}0.9154 \pm 0.0401 \\
(0.8114-0.9725)\end{array}$ & $\begin{array}{l}0.8908 \pm 0.0533 \\
(0.6870-0.9722)\end{array}$ & $\begin{array}{l}0.9213 \pm 0.407 \\
(0.8912-0.9810)\end{array}$ \\
\hline Time & $61 \min$ & $57.1 \mathrm{~min}$ & $64 \mathrm{~min}$ & $47.5 \mathrm{~min}$ & $66 \mathrm{~min}$ & $65.9 \mathrm{~min}$ \\
\hline
\end{tabular}

Itt: Iteration, SVM: Support Vector Machine 
Table 3: The effect of number of repetitions on time and segmentation accuracy and comparing different grouping methods in the right lung

\begin{tabular}{ccccccc} 
& Manual & SVM.manual & Dice & SVM- Dice & $\begin{array}{c}\text { Correlation } \\
\text { ratio }\end{array}$ & $\begin{array}{c}\text { SVM Correlation } \\
\text { ratio }\end{array}$ \\
\hline \multirow{2}{*}{ Itt $\mathbf{5 0}$} & $0.9481 \pm 0.0253$ & $0.9464 \pm 0.0274$ & $0.9494 \pm 0.0289$ & $0.9454 \pm 0.0292$ & $0.9372 \pm 0.0411$ & $0.9397 \pm 0.0313$ \\
& $(0.8647-0.9788)$ & $(0.8713-0.9807)$ & $(0.8155-0.9848)$ & $(90.8346-0.9846)$ & $(0.6422-0.9751)$ & $(0.7854-0.9818)$ \\
\hline Time & $23.3 \mathrm{~min}$ & $25.9 \mathrm{~min}$ & $22 \mathrm{~min}$ & $22 \mathrm{~min}$ & $23.9 \mathrm{~min}$ & $25.3 \mathrm{~min}$ \\
\hline \multirow{2}{*}{ Itt 70 } & $0.9485 \pm 0.0280$ & $0.9461 \pm 0.0292$ & $0.9483 \pm 0.0325$ & $0.9450 \pm 0.0330$ & $0.9386 \pm 0.0412$ & $0.9427 \pm 0.0271$ \\
& $(0.8548-0.9812)$ & $(0.8408-0.9816)$ & $(0.8083-0.9852)$ & $(0.8217-0.9837)$ & $(0.6416-0.9827)$ & $(0.8742-0.9819)$ \\
\hline Time & $27.6 \mathrm{~min}$ & $29.4 \mathrm{~min}$ & $27.4 \mathrm{~min}$ & $26 \mathrm{~min}$ & $27.8 \mathrm{~min}$ & $27.5 \mathrm{~min}$
\end{tabular}

Itt: Iteration, SVM: Support Vector Machine

were primarily related to the SVM trained by grouping through the Dice similarity coefficient and then the SVM method training with expert supervision. Moreover, this method is incredibly superior to the initial grouping methods. Thus, the best grouping method for training ASM code is the SVM- Dice method.

The accuracy of ASM segmentation results based on the SVM-Dice grouping for $150 \mathrm{im}$ ages of the tuberculosis database was obtained at $0.8833 \pm 0.0672$ and $0.9037 \pm 0.0548$, respectively, for left and right lungs. These values were $0.9412 \pm 0.0234$ and $0.9438 \pm$ 0.0374 , for left and right lungs for 100 images from the database with images of pulmonary nodes. According to these results, the method presented in this project has good accuracy for both groups of test data; however, a significant reduction in accuracy for tuberculosis images can be due to the low image quality and the need for a large amount of pre-processing before starting the segmentation method.

\section{Discussion}

ASM could be powerful to segment tissues like lungs by exploiting the prior data on the shape and gray-scale distribution [7] and the foremost challenge of segmentation with ASM is avoiding and reducing the model specified misclassification. In this paper, we have shown a method to customize ASM for segmenting lungs based on chest radiography images.

According to the results, the mean of detected nodules for the left and right lungs were $94.12 \pm 2.34 \%$ and $94.38 \pm 3.74 \%$, respectively. The differences in segmentation strategies, variability within the datasets, validation procedures, labeling and scoring strategies, different optimization methods, and the number of operation points led to the difficult definitive comparison. However, this study aims to identify published results using the JSRT data or a database of comparable difficulty for mitigating the variability factors [16-18]. The dataset used by Shiraishi et al. [19] is the largest one, employing 1000 cases. These data include the JRST data, but many other cases from different origins as well. The size and subtle distribution reported for the nodules in this database are sufficiently similar to those of the JRST subset to warrant a segmentation performance comparison. The RS-2000 was tested on a database of similar difficulty as the JSRT database and obtained an area $A_{z}$ score of 0.833 for the JSRT database and 0.835 for the database in Freedman et al. [20]. In this particular study, the RS-2000 system detected $66 \%$ of the nodules with on average 5 false positives per image. However, RS-2000 has proven its worth in over 10000 cases to obtain FDA approval, whereas our system is tested on the JSRT database with 100 nodule cases.

Wei et al. [17] reported a sensitivity of $80 \%$ 
for the JSRT database. However, this sensitivity was obtained using 202 uncorrelated features, this might be a problem as the system uses more features than the available number of true positive samples in the database, leading to a higher risk of overtraining the system. Coppini et al. [18] also used the JSRT database, and the sensitivity of their segmentation system was $60 \%$. The results of the previous studies showed a better accuracy for the proposed customized ASM in comparison with the traditional ASM [7]. In addition, segmentation with ASM along with SIFT features increases accuracy [21], due to the customization which better reflects the imaging features of chest radiography images around the lungs and better initialization. The performance is very close to the hybrid voting [7] which combines the strength of the best ASM, AAM, and pixel classification. The proposed ASM might improve the performance of hybrid voting if the proposed ASM is adopted for voting. According to the results from the current work, the yielded average accuracy is $94.25 \%$ for JSRT database.

The proposed strategy has also been compared with the thresholding-based method [22] to find better accuracy $(0.9425$ vs. 0.938$)$ and better shape preservation. Our results showed that the ASM segmentation method with presegmentation grouping could detect a higher number of nodules compared to previous studies. Furthermore, testing this method on tuberculosis radiographs also showed a high detection ratio, which may be related to our proposed approach. This proposed strategy could be useful for the customization of ASM to different images and could be an expected apparatus for a computer-aided finding of lung illnesses from chest radiography images.

\section{Conclusion}

The best method for grouping images into 3 general groups: healthy, diaphragm beating, and large heart, was SVM trained by Dice similarity coefficient grouping with 0.7956 accuracies in grouping images. Therefore, the SVM-Dice grouping method is suitable as a pre-processing step for images before ASM code, increasing the segmentation accuracy. In the current work, grouping images before segmentation by ASM resulted in increasing the segmentation accuracy. The code provided in this project can be used as a preliminary step to identify areas with tuberculosis or pulmonary nodules or cancers and measure the size and dimensions of the heart in diseases in which it is critical to examine cardiac changes using serial images of the patient to detect heard resizing.

\section{Authors' Contribution}

M. Dorri Giv carried out all steps of the study from conception to manuscript, drafting, and critical revision. M. Haghighi Borujeini participated in the conception and design of the study, analyzing and interpretation of data. D. Seifi Makrani participated in the acquisition of data, and critical revision of the manuscript. L. Dastranj carried out the critical revision of the manuscript, analyzing and interpreting of the data. M. Yadollahi participated in the conception and design of the study, critical revision of the manuscript, analyzing and interpretation of data. S. Semyari carried out the critical revision of the manuscript, analyzing and interpreting of data. M. Sadrnia participated in the conception and design of the study, and critical revision of the manuscript. Gh. Ataei carried out the critical revision of the manuscript, analyzing and interpreting of data. H. Riahi Madvar conceived of the design of the study, analyzing and interpretation of data, and critical revision of the manuscript. All authors read the final version of the manuscript and approved it.

\section{Ethical Approval}

The data was not extracted in real patients.

\section{Conflict of Interest}

None

\section{References}

1. Wu G, Zhang X, Luo S, Hu Q. Lung segmentation based on customized active shape model from digital radiography chest images. J Med Imaging Health Inform. 
2015;5(2):184-91. doi: 10.1166/jmihi.2015.1382.

2. Duncan JS, Ayache N. Medical image analysis: Progress over two decades and the challenges ahead. IEEE Trans Pattern Anal Mach Intell. 2000;22(1):85-106. doi: 10.1109/34.824822.

3. Nolden M, Zelzer S, Seitel A, Wald D, Müller M, Franz $\mathrm{AM}$, et al. The medical imaging interaction toolkit: challenges and advances. Int J Comput Assist Radiol Surg. 2013;8(4):607-20. doi: 10.1007/s11548-0130840-8. PubMed PMID: 23588509.

4. Armato III SG, Sensakovic WF. Automated lung segmentation for thoracic CT: impact on computeraided diagnosis1. Acad Radiol. 2004;11(9):1011-21. doi: 10.1016/j.acra.2004.06.005. PubMed PMID: 15350582.

5. Yepes-Calderon F, McComb JG. Manual Segmentation Errors in Medical Imaging Proposing a Reliable Gold Standard. International Conference on Applied Informatics; Cham: Springer; 2019. p. 230-41. doi: 10.1007/978-3-030-32475-9_17.

6. Akhil M, Aishwarya R, Lal V, Mahesh S. Comparison and evaluation of segmentation techniques for brain mri using Gold Standard. Indian J Sci Technol. 2016;9(46):1-5. doi: 10.17485/ijst/2016/ v9i46/106495.

7. Van Ginneken B, Stegmann MB, Loog M. Segmentation of anatomical structures in chest radiographs using supervised methods: a comparative study on a public database. Med Image Anal. 2006;10(1):19-40. doi: 10.1016/j.media.2005.02.002. PubMed PMID: 15919232.

8. Cootes TF, Edwards GJ, Taylor CJ. Comparing active shape models with active appearance models. BmvC. 1999:173-82. doi: 10.5244/C.13.18.

9. De Bruijne M, Van Ginneken B, Viergever MA, Niessen WJ. Adapting active shape models for 3D segmentation of tubular structures in medical images. Inf Process Med Imaging. 2003;18:136-47. doi: 10.1007/978-3-540-45087-0_12. PubMed PMID: 15344453.

10. Heimann T, Wolf I, Williams T, Meinzer HP. 3D active shape models using gradient descent optimization of description length. Inf Process Med Imaging. 2005;19:566-77. doi: 10.1007/11505730_47. PubMed PMID: 17354726.

11. De Nunzio G, Tommasi E, Agrusti A, Cataldo R, et al. Automatic lung segmentation in CT images with accurate handling of the hilar region. J Digit Imaging. 2011;24(1):11-27. doi: 10.1007/s10278-0099229-1. PubMed PMID: 19826872. PubMed PMCID: PMC3046791.

12. Zhou S, Cheng Y, Tamura S. Automated lung segmentation and smoothing techniques for inclusion of juxtapleural nodules and pulmonary vessels on chest CT images. Biomed Signal Process Control. 2014;13:6270. doi: 10.1016/j.bspc.2014.03.010.
13. Shi Y, Shen D. Hierarchical shape statistical model for segmentation of lung fields in chest radiographs. Med Image Comput Comput Assist Interv. 2008;11(Pt 1):417-24. doi: 10.1007/978-3-540-85988-8_50. PubMed PMID: 18979774.

14. Chen S, Suzuki K, MacMahon H. Development and evaluation of a computer-aided diagnostic scheme for lung nodule detection in chest radiographs by means of two-stage nodule enhancement with support vector classification. Med Phys. 2011;38(4):1844-58. doi: 10.1118/1.3561504. PubMed PMID: 21626918. PubMed PMCID: PMC3069992.

15. Van Ginneken B, Frangi AF, Staal JJ, Ter Haar Romeny $B M$, Viergever MA. Active shape model segmentation with optimal features. IEEE Trans Med Imaging. 2002;21(8):924-33. doi: 10.1109/TMI.2002.803121. PubMed PMID: 12472265.

16. Schilham AM, Van Ginneken B, Loog M. A computeraided diagnosis system for detection of lung nodules in chest radiographs with an evaluation on a public database. Med Image Anal. 2006;10(2):247-58. doi: 10.1016/j.media.2005.09.003. PubMed PMID: 16293441.

17. Wei J, Hagihara Y, Shimizu A, Kobatake H. Optimal image feature set for detecting lung nodules on chest X-ray images. CARS 2002 Computer Assisted Radiology and Surgery; Berlin, Heidelberg: Springer; 2002. p. 706-11. doi: 10.1007/978-3-642-56168-9_118.

18. Coppini G, Diciotti S, Falchini M, Villari N, Valli G. Neural networks for computer-aided diagnosis: detection of lung nodules in chest radiograms. IEEE Trans Inf Technol Biomed. 2003;7(4):344-57. doi: 10.1109/ titb.2003.821313. PubMed PMID: 15000360.

19. Shiraishi J, Li Q, Suzuki K, Engelmann R, Doi K. Computer-aided diagnostic scheme for the detection of lung nodules on chest radiographs: Localized search method based on anatomical classification. Med Phys. 2006;33(7):2642-53. doi: 10.1118/1.2208739. PubMed PMID: 16898468.

20. Freedman MT, Lo S-CB, Osicka T, Lure FYM, Xu X-W, Lin J, et al. Computer-aided detection of lung cancer on chest radiographs: effect of machine CAD false-positive locations on radiologists' behavior. Proceedings of the SPIE. 2002:1311-19. doi: 10.1117/12.467092.

21. Shi Y, Qi F, Xue Z, Chen L, Ito K, Matsuo H, et al. Segmenting lung fields in serial chest radiographs using both population-based and patient-specific shape statistics. IEEE Trans Med Imaging. 2008;27(4):48194. doi: 10.1109/TMI.2007.908130. PubMed PMID: 18390345.

22. Soleymanpour E, Pourreza HR. Fully automatic lung segmentation and rib suppression methods to improve nodule detection in chest radiographs. J Med Signals Sens. 2011;1(3):191-9. doi: 10.4103/22287477.95412. PubMed PMID: 22606675. PubMed PMCID: PMC3347229. 